\title{
CDK4 / 6 Inhibitor Palbociclib Combined with Radiotherapy in the Treatment of Cancer
}

\author{
Yasemin Benderli Cihan Cihan YB* \\ Kayseri Education and Research Hospital, Department of Radiation Oncology, Turkey \\ *Corresponding author: Yasemin Benderli Cihan, Kayseri Education and Research Hospital, Department of Radiation \\ Oncology, Sanayi District, Ataturk Boulevard, Hastane Street, No 78, 38010 Kocasinan, Kayseri, Turkey
}

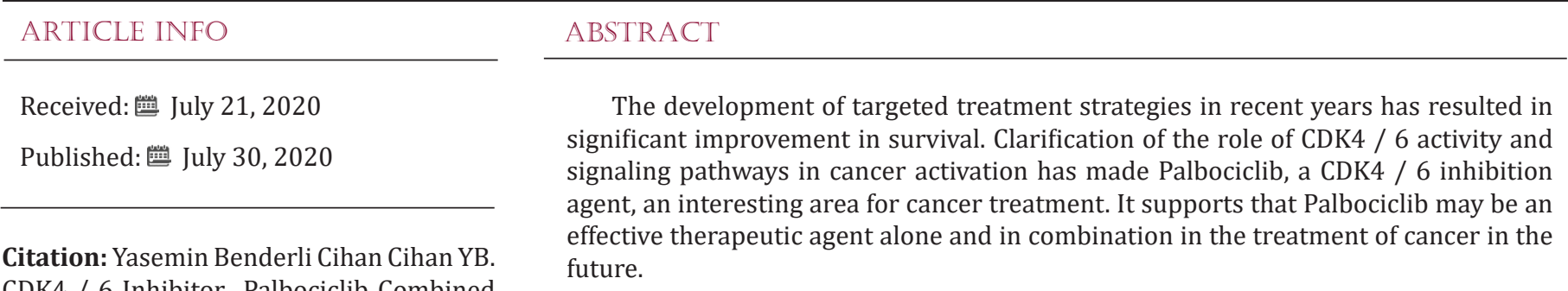
CDK4 / 6 Inhibitor Palbociclib Combined with Radiotherapy in the Treatment of Cancer. Biomed J Sci \& Tech Res 29(2)2020. BJSTR. MS.ID.004768.
Keywords: CDK4 / 6 Inhibitor; Palbociclib; Cancer; Radiotherapy

\section{Short Communication}

Cyclin D-cyclin dependent kynases (CDK4 / 6) is one of the key proteins involved in the G1-S phase of the cell cycle. CDK4 / 6 plays a role in the phosphorylation of retinoblastoma $(\mathrm{Rb})$ protein. This way it manages the G1-S phase. Disruption of this pathway and loss of coordination leads to uncontrolled cell proliferation and tumor growth. Disorders of this pathway have been shown in cancers such as pancreas, breast, lung, head and neck, and melanoma. CDK4 / 6 has become the target molecule in the treatment of advanced stage cancer and CDK4 / 6 inhibitors approved. Up to now 3 CDK4 / 6 inhibitors have been developed. These ara Palbociclib (PD-0332991), Ribociclib (LEE011) and Abemaciclib (LY2835219). Although all three agents are CDK4 / 6 inhibitors, effective doses and side effect profiles are different in treatment [13]. A cell cycle suppressor, Palbociclib, blocks the G1-S transition, stopping the cells at G0-G1. In addition, this agent has been shown in preclinical and clinical studies in $\mathrm{Rb}+$ cells by inhibiting cell proliferation. The effectiveness of Palbociclib in many types of cancers has been studied. Clinical response to the drug has been reported in the treatment of mantle cell lymphoma, liposarcoma and metastatic $\mathrm{Rb}+$ breast cancer, squamous cell lung cancer, neoadjuvant breast cancer and melanoma. Currently, studies investigating the effectiveness of various cancers are continuing [15]. In the PALOMA-1 study, patients with ER positive, HER2 negative postmenopausal advanced stage breast cancer were divided into groups taking Letrozole-Palbociclib and taking only Letrozole. Progression-free survival (PFS) was 20.2 months in the group treated with PFS and only 10.2 months in the group receiving Letrozole. Palbociclib ER was approved by the FDA in 2015 for use in HER2 negative postmenopausal advanced breast cancer. Phase 3 PALOMA-2 study is still continuing [1-3]. The efficacy of Fulvestrant and Palbociclib combination therapy was investigated by phase 3 PALOMA-3 study. In this study, survival was higher in combination therapy (3,8 months and 9,2 months, respectively). Studies on the use of neoadjuvant in breast cancer are continuing [1]. The efficacy of Palbociclib over 15 cancer treatments has been investigated. Research on the preclinical and clinical use of other cancers is also underway [2-7]. In the phase 2 study presented in ASCO 2018, the efficacy and safety of Palbociclib (Ibrance) in the treatment of metastatic or recurrent, non-HPV-associated head and neck cancers were examined. While median overall survival was 6 months with cetuximab alone, Palbociclib added 12,1 months. As a result, median overall survival at 12,1 months was reported to be the longest reported time for platinum-resistant recurrentmetastatic squamous cell carcinoma. In this study, Palbociclib which is commonly used in the treatment of neck cancers, has reversed the resistance of cetuximab in resistance against EGFR inhibitors 
such as cetuximab (Erbitux) [7]. As a side effect of palbociclib, a decrease in the number of neutrophils from blood immune cells has been observed, but no serious problems have arisen in patients due to this side effect. This phase has been shown to be a reliable agent in terms of side effects.

Palbociclib drugs began to be used with radiotherapy (RT) after the start of combined use. Phase 1 studies are currently in progress. Tao et al in vivo and in vitro in a phase 1 study with the addition of MEK inhibitor (Trametinib) and radiation CDK4 / 6 inhibitor (Palbociclib) reported that sensitizing KRAS-mutant NSCLCs [4] Hashizume et al, in the phase 1 study, human atypical teratoid rhabdoid tumor and glioblastoma in combination with radiation for the treatment of Palbociclib has been reported to show additive effect [8]. In another in vitro study, the use of Palbociclib combined with RT in GMB cells has been reported to have a synergistic effect [7]. For the first time, the combination of Palbociclib and radiotherapy was reported in the preliminary results of a clinical study by Hans et al. In this study, 5 patients with metastatic breast cancer underwent a daily dose of $125 \mathrm{mg}$ with Palbociclib, with Fulvestrant 500mg every 28 days at the same time. It was stated that there is no increased toxicity in the combined use and it can be used safely in symptomatic patients [5]. As a result, the development of targeted treatment strategies in recent years has resulted in significant improvement in survival. After clarifying the role of CDK4 / 6 activity and signaling pathways in cancer activation, the CDK4 / 6 inhibiting agent, Palbociclib, has become an area of interest for cancer treatment. It supports that Palbociclib may be an effective therapeutic agent alone and in combination in the treatment of cancer in the future. Large-scale preclinical and clinical studies are needed.

\section{References}

1. Hamilton E, Infante JR (2016) Targeting CDK4/6 in patients with cancer. Cancer Treatment Reviews 201(45): 129-138.

2. Spring L, Bardia A, Modi S (2016) Targeting the cyclin D-cyclindependent kinase (CDK) 4/6- retinoblastoma pathway with selective CDK 4/6 inhibitors in hormone receptor-positive breast cancer: rationale, current status, and future directions. Discovery Medicine 21(113): 65-74

3. Ban M, Miše BP, Majić A, Dražić I, Vrdoljak E (2018) Efficacy and safety of palbociclib in heavily pretreated patients with HR+/HER2- metastatic breast cancer. Future Oncol 14(6): 537-544.

4. Tao Z, Le Blanc JM, Wang C, Zhan T, Zhuang H, et al. (2016) Coadministration of Trametinib and Palbociclib Radiosensitizes KRASMutant Non-Small Cell Lung Cancers In Vitro and In Vivo. Clin Cancer Res 22(1): 122-133

5. Hans S, Cottu P, Kirova YM (2018) Preliminary results of the association of Palbociclib and radiotherapy in metastatic breast cancer patients. Radiother Oncol 126(1): 181.

6. Whittaker S, Madani D, Joshi S, Chung SA, Johns T, et al. (2017) Combination of palbociclib and radiotherapy for glioblastoma. Cell Death Discov 3: 17033

7. Douglas Adkins, Peter John Oppelt, Jessica C Ley, Kathryn Trinkaus, Prakash C. Neupane, et al. Multicenter phase II trial of palbociclib, a selective cyclin dependent kinase (CDK) 4/6 inhibitor, and cetuximab in platinum-resistant HPV unrelated $(-)$ recurrent/metastatic head and neck squamous cell carcinoma (RM HNSCC).2018 ASCO Annual Meeting - Abstract No: 6008

8. Hashizume R, Zhang A, Mueller S, Prados MD, Lulla RR, et al. (2016) Inhibition of DNA damage repair by the CDK4/6 inhibitor palbociclib delays irradiated intracranial atypical teratoid rhabdoid tumor and glioblastoma xenograft regrowth. Neuro Oncol 18(11): 1519-1528.
ISSN: 2574-1241

\section{DOI: 10.26717 /BISTR.2020.29.004768}

Yasemin Benderli Cihan Cihan YB. Biomed J Sci \& Tech Res

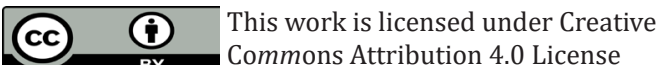

Submission Link: https://biomedres.us/submit-manuscript.php

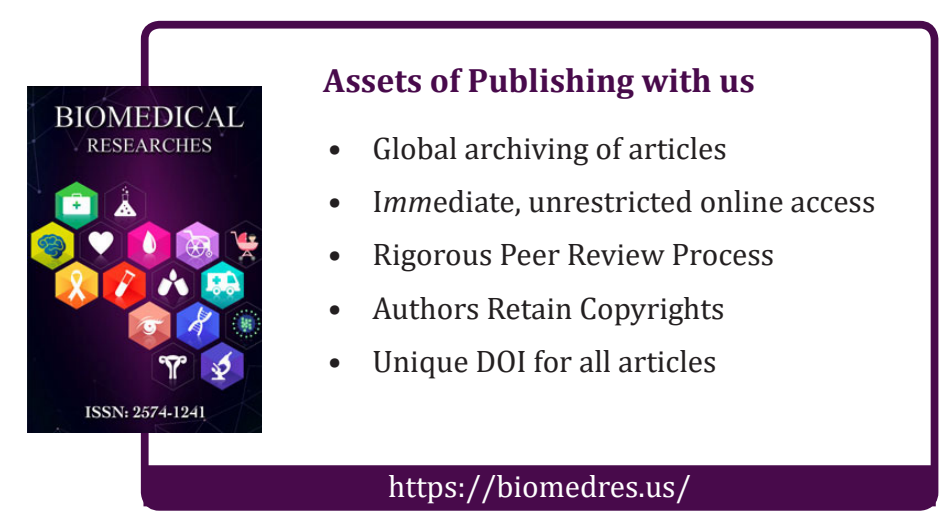

